Editorial

\title{
Socioeconomic disadvantages in childhood and metabolic syndrome risk: A call to action?
}

\author{
Ana Teresa Timóteo \\ Cardiology Department University Centre, Santa Marta Hospital, Lisbon, Portugal \\ NOVA Medical School, Lisbon, Portugal
}

\section{A R T I C L E I N F O}

Article history:

Received 3 August 2018

Accepted 8 August 2018

Available online 11 August 2018

Cardiovascular diseases are the leading cause of death in developed countries and in Europe, accounting for more than 20\% of deaths [1]. Metabolic syndrome, which is defined by an aggregation of several risk factors, namely hypertension, high triglycerides, low HDL-cholesterol, glucose intolerance/diabetes and increased waist circumference, is a well-established risk factor for cardiovascular diseases and several studies and meta-analysis performed in the last decade have consistently confirmed that association, particularly in primary prevention [2-5].

Previous studies demonstrated that low socioeconomic status in adults (low educational level, low income, holding a low-status job or living in a poor residential area) is closely related to psychosocial risk factors such as chronic stress at work and in family life, depression, anxiety, hostility and socially isolation/lack of social support, that cluster in individuals and groups and increases the risk of coronary artery disease, worsening prognosis [6]. Another link is through unhealthy lifestyle, such as more frequent smoking, unhealthy food choices, less physical activity and low adherence to behaviour changes recommendations. For that reason, assessment of psychosocial risk factors is of the upmost importance to consider as risk modifiers in cardiovascular risk prediction and prevention [6].

Socioeconomic inequalities in children and adolescents are increasing and it accompanies the increase in obesity prevalence. This is particularly clear in a study that compared several cohorts. In cohorts from 1946 to 1970, patients from low socioeconomic background presented lower weight in childhood and adolescence [7]. However, in a later cohort, from 2001, low socioeconomic status was associated with higher weight. In both cohorts, the difference in weight between socioeconomic status became larger from childhood to adolescence. This study showed that obesity presence in low socioeconomic communities is a more recent phenomenon. There is also a different direction according to gender, with obese men being predominately from higher socioeconomic status in childhood and obese women from lower socioeconomic status suggesting different impact according to gender $[8,9]$.
The paper published in the present number of International Journal of Cardiology by (Iguacel, et al.) studied a group of children included in The Identification and Prevention of Dietary- and Lifestyle-induced Health Effects in Children and Infants (IDEFICS) study in European children [10]. Parental questionnaires were applied at baseline and again two years later and consisted of information regarding education, income, social network, family structure, origin and employment status to assess parental socioeconomic disadvantages. Children (aged 2-10 years) were examined for vital signs, anthropometric data and blood testing for metabolic syndrome scoring. A well-being score and lifestyle indicators (fruit and vegetables consumption, physical activity and screen time) questionnaire were also applied to children. The authors concluded that children from low-income and non-traditional families and those whose parents were unemployed and who accumulated more than three disadvantages showed a higher metabolic syndrome score. However, in the fully adjusted models with additional adjustment for well-being score and lifestyle indicators, estimates were attenuated.

The study has some limitations as pointed out by the authors. Probably the most important limitation is the sample size. IDEFICS study included 16,229 children at baseline and 11,041 children in the second evaluation. However, in the present analysis, only 2401 children were effectively included. This is a major drop-out, with only approximately $15 \%$ of the initial sample included in this analysis. This smaller sample might be the cause for some bias, particularly due to the fact that this sample is restricted only to patients with specific information and some socioeconomic disadvantages are represented by small numbers of individuals, such as 130 unemployed parents, 235 migrants and 138 with 3-6 disadvantages. For that reason, results must be interpreted with some caution but they point to an important direction that should be studied in larger samples. Another limitation, although possibly less significant, is the use of point-of-care analyzers to assess lipid and glucose homeostasis data that are not as accurate as samples from peripheral venous blood.

There are two important conclusions from the present study. Firstly, that the main socioeconomic disadvantages in childhood associated with high metabolic syndrome risk are being from non-traditional families and parent's employment status and also that individuals with several accumulated disadvantages have an increased risk. The second major conclusion is that although a child is living in the presence of some form of socioeconomic disadvantage, the impact in metabolic 
syndrome risk can be significantly attenuated by lifestyle factors and children's well-being. This is particularly important because it can open a window of opportunity that should not be missed. Regulators, local authorities and the community should implement major interventions to improve children's well-being and lifestyle both in school and at home. These interventions should be implemented in close association with social workers and health care professionals (doctors, nurses, nutritionists, psychologists, and others) promoting healthy weight through the design of neighbourhoods that support and enhance the effect of physical activity. Education measures, such as healthy eating are also very important early in school and for parents. It is important to start all those interventions early in life, to prevent type 2 diabetes and cardiometabolic diseases.

\section{Conflict of interest}

The authors report no relationships that could be construed as a conflict of interest.

\section{References}

[1] N. Townsend, L. Wilson, P. Bhatnagar, et al., Cardiovascular diseased in Europe: epidemiological update 2016, Eur. Heart J. 37 (2016) 3232-3245.
[2] A.S. Gami, B.J. Witt, D.E. Howard, et al., Metabolic syndrome and risk of incident cardiovascular events and death: a systematic review and meta-analysis of longitudinal studies, J. Am. Coll. Cardiol. 49 (2007) 403-414.

[3] S. Motillo, K.B. Filion, J. Genest, et al., The metabolic syndrome and cardiovascular risk: a systematic review and meta-analysis, J. Am. Coll. Cardiol. 56 (2010) 1113-1132.

[4] S. Novo, A. Peritore, F.P. Guameri, et al., Metabolic syndrome predicts cardio and cerebrovascular events in twenty years follow-up. A prospective study, Atherosclerosis 223 (2012) 468-472.

[5] M. Fiuza, N. Cortez-Dias, S. Martins, et al., (on behalf of VALSIM study investigators). Metabolic syndrome in Portugal: prevalence and implications in cardiovascular risk results from VALSIM study, Rev. Port. Cardiol. 27 (2008) 1495-1529.

[6] M.F. Piepoli, A.W. Hoes, S. Agewall, et al., 2015 European guidelines on cardiovascular disease prevention in clinical practice, Eur. Heart J. 37 (2016) 2315-2381.

[7] D. Bann, W. Johnson, L. Li, D. Kuh, R. Hardy, Socioeconomic inequalities in childhood and adolescence body mass index, weight, and height from 1953 to 2005: an analysis of four longitudinal, observational, British birth cohort studies, Lancet Public Health 3 (2018) e194-e203.

[8] K.J.P. Wagner, J.L.D. Bastos, A. Navarro, D.A. Gonzalez-Chica, A.F. Boing, Socioeconomic status in childhood and obesity in adults: a population-based study, Rev. Saude Publica 52 (2018) 15.

[9] T.Z. Nobari, S.E. Whaley, M.L. Prelip, C.M. Crespi, M.C. Wang, Trends in socioeconomic disparities in obesity prevalence among low-income children aged 2-4 years in Los Angeles County, 2003-2014, Child Obes. 14 (2018) 248-258.

[10] I. Iguacel, N. Michels, W. Ahrens, et al., Prospective associations between socioeconomically disadvantaged groups and metabolic syndrome risk in European children. Results from the IDEFICS study, Int. J. Cardiol. (2018) https://doi.org/10.1016/j. ijcard.2018.07.053. 